\title{
Configurable Modulator for Pure Phase Modulation
}

\author{
Hong Deng and Wim Bogaerts \\ Photonics Research Group, Department of Information Technology (INTEC) \\ Ghent University - IMEC, Gent, Belgium \\ E-mail: hong.deng@ugent.be
}

\begin{abstract}
We present a configurable silicon modulator for pure phase modulation, implemented as a Mach-Zehnder interferometer with a PN carrier depletion modulator (CDM) and tunable couplers. The spurious intensity modulation introduced by the CDM is compensated by tuning the splitting ratios of the couplers and the phase delay between the arms. In this report, we present the simulation results of this device.
\end{abstract}

Index Terms-Reconfigurable photonic circuits, modulators, phase modulators

\section{INTRODUCTION}

Silicon photonics is one of the most promising photonics integrating platforms, useful for a variety of applications. One key component is an electro-optic phase modulator [1]. Silicon carrier-depletion modulators (CDM), realized by embedding a PN junction in a silicon waveguide, are the most common solution for high-speed modulation. A reverse bias over the PN junction changes the refractive index of the waveguide, introducing a phase shift.

However, CDMs suffer from spurious intensity modulation, as the propagation loss also depends on the carrier density. A thermo-optic phase shifter can achieve pure phase modulation, with a limited bandwidth of a few MHz. Here, we report a method to compensate the spurious intensity modulation by embedding the CDM into a configurable circuit. Simulation results show that we can suppress the intensity modulation down to $0.1 \mathrm{~dB}$ within a phase change of $0.4 \pi$.

\section{PRinciple AND Simulation}

We implement the configurable modulator circuit, shown in Fig. 1, as an MZI with using two tunable couplers (TC). The splitting ratio of the TCs can be tuned from $0 \%$ to $100 \%$ [2]. One arm contains a CDM, while the other arm contains a phase shifter (PS) to tune the phase delay between the two arms.

The transmission of the MZI is the sum of complex phasors at the end of arms. The CDM will rotate the phasor of the upper arm and also increase its amplitude (with a reversebiased PN junction); the phasor of the other arm will stay the same. This changing phase delay will therefore change the sum of both phasors. By adjusting their relative amplitude and angle between them, the spurious intensity modulation can be minimized and pure phase modulation can be obtained.

We simulated this circuit, using the $V_{\pi} \cdot L_{\pi}$ and voltagedependent loss of the CDM as described in [3]. Figure 2a shows the simulated intensity modulation (Max/Min) at the output of the circuit as function of the splitting ratio $(\mathrm{TC} 1=\mathrm{TC} 2)$ and the phase delay, which is the lower the better. Fig. $2 \mathrm{~b}$ shows the

This work has received funding from the European Research Council through grant agreement No 725555 (PhotonicSWARM).

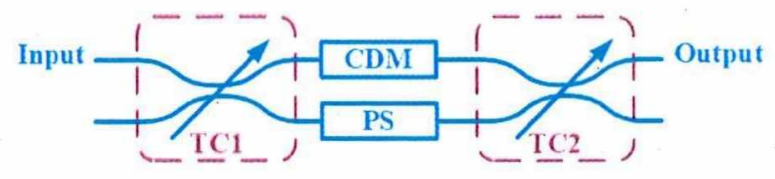

Fig. 1. Schematic of the reconfigurable modulator. PS: phase shifter; PM: phase modulator; TC: tunable coupler.

voltage response at the operation point highlighted in Fig. 2a. The CDM introduces $1.25 \mathrm{~dB}$ intensity modulation and a $0.4 \pi$ phase modulation with a voltage of $6 \mathrm{~V}$, while the output of the configurable modulator only has $0.1 \mathrm{~dB}$ intensity modulation and almost the same $0.4 \pi$ phase modulation. The trade-off is that the circuit does introduce around $0.5 \mathrm{~dB}$ additional loss.

\section{CONCLUSION}

We reported a configurable modulator for pure phase modulation. Simulation results showed that the $1.25 \mathrm{~dB}$ spurious intensity modulation can be suppressed down to $0.1 \mathrm{~dB}$ within a phase range of $0.4 \pi$.

\section{REFERENCES}

[1] G. T. Reed, G. Mashanovich, F. Y. Gardes, and D. J. Thomson, "Silicon optical modulators," Nat. Photonics, vol.4, no.8, pp. 518526, Aug. 2010. [2] K. Suzuki, G. Cong, K. Tanizawa, SH. Kim, K. Ikeda, S. Namiki, H. Kawashima, "Ultra-high-extinction-ratio 22 silicon optical switch with variable splitter," Opt. Express., vol. 23, no. 7, pp.9086-9092, Apr. 2015.

[3] H. Yu, M. Pantouvaki, J. Van Campenhout, D. Korn, K. Komorowska, P. Dumon, Y. Li, P. Verheyen, P. Absil, L. Alloatti, D. Hillerkuss, "Performance tradeoff between lateral and interdigitated doping patterns for high speed carrier-depletion based silicon modulators," Opt. Express., vol. 20, no. 12, pp. 12926-12938, Jun. 2012.
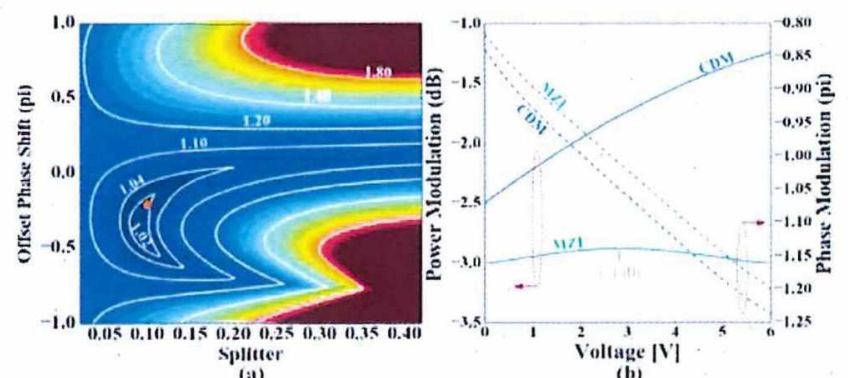

Fig. 2. Simulation results. (a) spurious amplitude modulation for different coupling ratios and phase shifts, (b) Voltage response for the optimal configuration. 


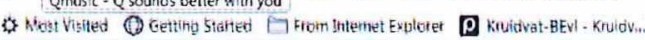

Corferencés is 2019 Phetonics North (PW) $\quad$ O

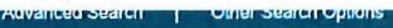

\section{Configurable Modulator for Pure Phase Modulation}

Publisher: IEEE

2 Author(s) Hong Deng, Wim Bogaerts Vlew All Authors

18

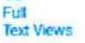

回

Abstract

Abstract:

Document Sections

We present a configurable silicon modulator for pure phase modulation. implemented as a Mach-Zehnder

1. Introduction interferometer with a PN carrier depletion modulator (CDM) and tunable couplers. The spurious intensity

II Principle and Simulation modulation introduced by the CDM is compensated by tuning the splitting ratios of the couplers and the

II. Conclusion

Published in: 2019 Photonics North (PN)

Authors

Date of Conference: 21-23 May 2019

Date Added to IEEE Xplore: 29 August 2019

Figures

References

$\checkmark$ ISBN Information:

Electronic ISBN: $978-1-7281-3738-4$

Print on Demand(POD) ISBN:

978-1-7281-3739-1

INSPEC Accession Number: 19024719

DOI: 10.1109/PN.2019.8819529

Publisher: IEEE

Conference Location: Quebec City, QC, Canada, Canada

Advertsement

Keywords

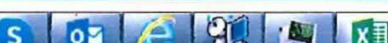

More Like This

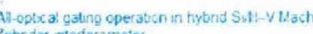

hatum Prosendide and Related Waenals

Pubbsred 2011

Sticon Wach Zerinder interterameter modviator wh th PANA data modut ation at $64 \mathrm{~Gb}$

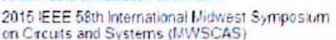
Putlsist 2015

Vievi Itore

Top Organizations with Patents on Top Organizations with Patents on
Technologies Mentioned in This Arlicle 